\title{
Gravitational Collapse on the Brane: A No-Go Theorem
}

\author{
Marco Bruni, Cristiano Germani, and Roy Maartens \\ Relativity and Cosmology Group, School of Computer Science and Mathematics, Portsmouth University, \\ Portsmouth PO1 2EG, United Kingdom \\ (Received 3 August 2001; published 13 November 2001)
}

\begin{abstract}
We investigate how braneworld gravity affects gravitational collapse and black hole formation by studying Oppenheimer-Snyder-like collapse on a Randall-Sundrum-type brane. Without making any assumptions about the bulk, we prove a no-go theorem: the exterior spacetime on the brane cannot be static, which is in stark contrast with general relativity. We also consider the role of Kaluza-Klein energy density in collapse, using a toy model.
\end{abstract}

DOI: $10.1103 /$ PhysRevLett.87.231302

PACS numbers: $04.50 .+\mathrm{h}, 11.10 . \mathrm{Kk}, 98.80 . \mathrm{Cq}$

The study of gravitational collapse in general relativity (GR) is fundamental to understanding the behavior of the theory at high energies. The Oppenheimer-Snyder (OS) model still provides a paradigmatic example that serves as a good qualitative guide to the general collapse problem in GR. It can be solved analytically, as it simply assumes a collapsing homogeneous dust cloud of finite mass and radius, described by a Robertson-Walker metric and surrounded by a vacuum exterior. In GR, this exterior is necessarily static and given by the Schwarzschild solution [1]. In other theories of gravity that differ from GR at high energies, it is natural to look for similar examples. Braneworld scenarios of the Randall-Sundrum type [2,3] lead to modified Einstein equations as the effective 4D field equations on the brane. In this Letter we analyze an OS-like collapse in this setting, in order to shed light on some fundamental differences between collapse in GR and on the brane.

In string theory and $M$ theory, which may provide a route towards quantum gravity, gravity is a truly higherdimensional interaction, which becomes effectively 4D at low enough energies. Simple braneworld models inspired by these theories describe the observable universe as a 3-brane boundary of a 4D space (the bulk), with matter fields confined on the brane. Gravity propagates in all four spatial dimensions but must be localized near the brane at low energies, in order to reproduce the successful predictions of GR. This is most obviously achieved via a small compact extra dimension, as in Kaluza-Klein (KK) theories, but the Randall-Sundrum model [2] localizes gravity by the curvature of the bulk, even with a noncompact extra dimension. The bulk metric, which is anti-de Sitter $\left(\mathrm{AdS}_{5}\right)$, satisfies the five-dimensional Einstein equations with negative cosmological constant. Their model, with vacuum Minkowski brane, has been generalized to allow for arbitrary energy-momentum tensor on the brane, and the effective field equations on the brane are modified Einstein equations [3].

Perturbative analysis of the gravitational field due to a compact source on the brane has been performed $[2,4,5]$. In particular, in the weak-field limit there is a $5 \mathrm{D}$ correction to the Newtonian potential on the brane which to leading order gives

$$
\Phi=(G M / r)\left[1+2 \ell^{2} / 3 r^{2}\right],
$$

where $\ell$ is the curvature scale of $\mathrm{AdS}_{5}$. Brane solutions of static black hole and stellar exteriors with five-dimensional corrections to the Schwarzschild metric have been found [6,7], but the bulk metric for these solutions has not been found. The Schwarzschild black string bulk metric has a Schwarzschild black hole on the brane but cannot describe the end state of gravitational collapse [8]. Numerical integration into the bulk, starting from static black hole solutions on the brane, is plagued with difficulties [9]. In summary, very little is known about astrophysical black holes and stars on the brane, even in the static case. Even less is known about astrophysical gravitational collapse on the brane to a black hole.

Braneworld gravitational collapse is complicated by a number of factors. The confinement of matter to the brane, while the gravitational field can access the extra dimension, is at the root of the difficulties relative to Einstein's theory, and this is compounded by the gravitational interaction between the brane and the bulk. Matching conditions on the brane are more complicated to implement [7], and one also has to impose regularity and asymptotic conditions on the bulk, and it is not obvious what these should be.

In GR, the OS model of collapsing dust has a RobertsonWalker interior matched to a Schwarzschild exterior. We show that even this simplest case is much more complicated on the brane. However, it does have a striking new property, which may be part of the generic collapse problem on the brane. The exterior is not Schwarzschild, nor could we expect it to be, as discussed above, but the exterior is not even static, as shown by our no-go theorem. The reason for this lies in the nature of the braneworld modifications to GR.

The field equations on a generalized Randall-Sundrum brane are [3]

$$
G_{\mu \nu}=-\Lambda g_{\mu \nu}+8 \pi G T_{\mu \nu}+48 \pi G S_{\mu \nu} / \lambda-\mathcal{E}_{\mu \nu},
$$


where $\lambda$ is the brane tension $\left(\lambda>10^{8} \mathrm{GeV}^{4}\right)$ and the brane cosmological constant $\Lambda$ has contributions from the tension and the bulk cosmological constant. The tensor $S_{\mu \nu}$ is quadratic in the energy-momentum tensor $T_{\mu \nu}$, and $\mathcal{E}_{\mu \nu}$ is the projection of the bulk Weyl tensor. The energy-momentum tensor satisfies the usual conservation equations, and the Bianchi identities on the brane then imply a "conservation" equation for the trace-free $\mathcal{E}_{\mu \nu}$ :

$\nabla^{\nu} T_{\mu \nu}=0, \quad \nabla^{\nu} \mathcal{E}_{\mu \nu}=48 \pi G \nabla^{\nu} S_{\mu \nu} / \lambda, \quad \mathcal{E}_{\mu}^{\mu}=0$.

The general $1+3$ form of these equations is given in [10]. Five-dimensional corrections to the field equations of GR are of two types $[3,10]$.

(1) High-energy corrections, given by $S_{\mu \nu}$, arise from the extrinsic curvature of the brane and increase the effective density and pressure of collapsing matter. In particular, this means that the effective pressure does not in general vanish at the surface, changing the nature of the matching conditions on the brane [7]. Gravitational collapse inevitably produces energies high enough to make these corrections significant.

(2) KK corrections, given by $\mathcal{E}_{\mu \nu}$, arise from $5 \mathrm{D}$ graviton stresses and are constrained by Eq. (3). In the linearized regime they are known as massive KK modes of the graviton [2]; in general, they are a signature of nonlinear KK modes in the bulk. For brane-bound observers, these stresses are nonlocal: local density inhomogeneities on the brane generate Weyl curvature in the bulk that "backreacts" nonlocally on the brane [10]. Even in the absence of matter, $\mathcal{E}_{\mu \nu}$ may be nonzero (provided that $\nabla^{\nu} \mathcal{E}_{\mu \nu}=0$ ), since there may be 5D Weyl curvature in the bulk, e.g., sourced by a bulk black hole, as in cosmological braneworld models [11,12]. The KK stresses further complicate the matching problem on the brane, since they in general contribute to the effective radial pressure at the surface.

Equations (2) and (3) are the complete set of equations on the brane. They are not closed, since $\mathcal{E}_{\mu \nu}$ contains 5D degrees of freedom that cannot be determined on the brane. A further set of 5D equations [3] makes up the full closed system. However, using only the 4D projected equations, we prove a no-go theorem valid for the full 5D problem: given the standard matching conditions on the brane, the exterior of a collapsing dust cloud cannot be static. We are not able to determine the nonstatic exterior metric, but we expect on general physical grounds that the nonstatic behavior will be transient, so that the exterior tends to a static form.

The collapsing region in general contains dust and also energy density on the brane from KK stresses in the bulk (this is called "dark radiation" in cosmology [11,12]). We show that in the extreme case where there is no matter but only collapsing homogeneous KK energy density, there is a unique exterior which is static for physically reasonable values of the parameters. Since there is no matter on the brane to generate KK stresses, the KK energy density on the brane must arise from bulk Weyl curvature. In this case, the bulk could be pathological. The collapsing KK energy density can either bounce or form a black hole with a 5D gravitational potential, and the exterior is of the Weyl-charged de Sitter type (given in [6] for $\Lambda=0$ ), but with no mass.

The collapse region has a Robertson-Walker metric

$$
d s^{2}=-d \tau^{2}+a(\tau)^{2}\left(1+\frac{1}{4} k r^{2}\right)^{-2}\left[d r^{2}+r^{2} d \Omega^{2}\right] .
$$

The modified Friedmann equation from Eq. (2) is [13]

$$
\dot{a}^{2} / a^{2}=\frac{8}{3} \pi G \rho(1+\rho / 2 \lambda)+C / \lambda a^{4}-k / a^{2}+\frac{1}{3} \Lambda,
$$

where the KK constant $C$ is fixed by the bulk Weyl curvature (for a cosmological Friedmann brane, $C$ is proportional to the mass of a black hole in the bulk [11,12]). The $\rho^{2}$ term, which is significant for $\rho \geq \lambda$, is the high-energy correction term, following from $S_{\mu \nu}$. Standard Friedmann evolution is regained in the limit $\lambda^{-1} \rightarrow 0$. Equation (3) implies $\rho=\rho_{0}\left(a_{0} / a\right)^{3}$, where $a_{0}$ is the epoch when the cloud started to collapse. The proper radius from the center of the cloud is $R(\tau)=r a(\tau) /\left(1+\frac{1}{4} k r^{2}\right)$. The collapsing boundary surface $\Sigma$ is given in the interior comoving coordinates as a free-fall surface, i.e., $r=r_{0}=$ const, so that $R_{\Sigma}(\tau)=r_{0} a(\tau) /\left(1+\frac{1}{4} k r_{0}^{2}\right)$.

We can rewrite the modified Friedmann equation on the interior side of $\Sigma$ as

$$
\begin{aligned}
\dot{R}^{2}= & 2 G M / R+3 G M^{2} / 4 \pi \lambda R^{4}+Q / \lambda R^{2} \\
& +E+\Lambda / 3 R^{2},
\end{aligned}
$$

where the "physical mass" $M$ (total energy per proper star volume) and the total "tidal charge" $Q$ are

$$
\begin{aligned}
M & =\frac{4}{3} \pi a_{0}^{3} r_{0}^{3} \rho_{0}\left(1+\frac{1}{4} k r_{0}^{2}\right)^{-3}, \\
Q & =C r_{0}^{4}\left(1+\frac{1}{4} k r_{0}^{2}\right)^{-4},
\end{aligned}
$$

and the "energy" per unit mass is given by

$$
E=-k r_{0}^{2}\left(1+\frac{1}{4} k r_{0}^{2}\right)^{-2}>-1 .
$$

Now we assume that the exterior is static and satisfies the standard 4D junction conditions. Then we check whether this exterior is physical by imposing the modified Einstein equations (2) for vacuum, i.e., for $T_{\mu \nu}=0=S_{\mu \nu}$. The standard 4D Israel matching conditions, which we assume hold on the brane, require that the metric and the extrinsic curvature of $\Sigma$ be continuous. The extrinsic curvature is continuous if the metric is continuous and if $\dot{R}$ is continuous [1]. We therefore need to match the metrics and $\dot{R}$ across $\Sigma$.

The most general static spherical metric that could match the interior metric on $\Sigma$ is

$$
\begin{aligned}
d s^{2} & =-F(R)^{2} A(R) d t^{2}+d R^{2} / A(R)+R^{2} d \Omega^{2}, \\
A(R) & =1-2 G m(R) / R .
\end{aligned}
$$


We need two conditions to determine the functions $F(R)$ and $m(R)$. Now $\Sigma$ is a freely falling surface in both metrics, and the radial geodesic equation for the exterior metric gives $\dot{R}^{2}=-A(R)+\tilde{E} / F(R)^{2}$, where $\tilde{E}$ is a constant and the dot denotes a proper time derivative, as above. Comparing this with Eq. (6) gives one condition. The second condition is easier to derive if we change to null coordinates. The exterior static metric, with $d v=d t+$ $d R /[F(1-2 G m / R)]$, becomes

$$
d s^{2}=-F^{2} A d v^{2}+2 F d v d R+R^{2} d \Omega^{2} .
$$

The interior Robertson-Walker metric takes the form [14]

$$
\begin{aligned}
d s^{2}= & -\tau_{, v}^{2}\left[1-\left(k+\dot{a}^{2}\right) R^{2} / a^{2}\right] d v^{2} /\left(1-k R^{2} / a^{2}\right) \\
& +2 \tau_{, v} d v d R / \sqrt{1-k R^{2} / a^{2}}+R^{2} d \Omega^{2},
\end{aligned}
$$

where $d \tau=\tau_{, v} d v+\left(1+\frac{1}{4} k r^{2}\right) d R /\left[r \dot{a}-1+\frac{1}{4} k r^{2}\right]$. Comparing Eqs. (10) and (11) on $\Sigma$ gives the second condition. The two conditions together imply that $F$ is a constant, which we can take as $F(R)=1$ without loss of generality (choosing $\tilde{E}=E+1$ ), and that

$$
m(R)=M+3 M^{2} / 8 \pi \lambda R^{3}+Q / 2 G \lambda R+\Lambda R^{3} / 6 G .
$$

In the limit $\lambda^{-1} \rightarrow 0$, we recover the $4 \mathrm{D}$ GR Schwarzschild-de Sitter solution. However, we note that the above form of $m(R)$ violates the weak-field perturbative limit in Eq. (1), and this is a symptom of the problem with a static exterior. Equations (9) and (12) imply that the brane Ricci scalar is

$$
R_{\mu}^{\mu}=4 \Lambda+9 G M^{2} / 2 \pi \lambda R^{6} .
$$

However, Eq. (2) for a vacuum exterior implies

$$
R_{\mu \nu}=\Lambda g_{\mu \nu}-\mathcal{E}_{\mu \nu}, \quad R_{\mu}^{\mu}=4 \Lambda .
$$

Comparing with Eq. (13), we see that a static exterior is possible only if $M / \lambda=0$. This is obviously satisfied in the GR limit, but in the braneworld, collapsing homogeneous and isotropic dust leads to a nonstatic exterior. We emphasize that this no-go result does not require any assumptions on the nature of the bulk spacetime.

The one case that escapes the no-go theorem is $M=0$. In GR, $M=0$ would lead to vacuum throughout the spacetime, but in the braneworld, there is the tidal KK stress on the brane, i.e., the $Q$ term in Eq. (6). The possibility of black holes forming from KK energy density was suggested in [6]. The dynamics of a Friedmann universe (i.e., without exterior), containing no matter but only KK energy density ("dark radiation") has been considered in [12]. In that case, there is a black hole in the Schwarzschild- $\mathrm{AdS}_{5}$ bulk, which sources the KK energy density. Growth in the KK energy density corresponds to the black hole and brane moving closer together; a singularity on the brane can arise if the black hole meets the brane. Here we investigate the collapse of a bound region of homogeneous KK energy density within an inhomogeneous exterior. It is not clear whether the bulk black hole model may be modified to describe this case, and we do not know what the bulk metric is. However, we know that there must be 5D Weyl curvature in the bulk and that the bulk could be pathological, with a more severe singularity than Schwarzschild-AdS S $_{5}$. Even though such a bulk would be unphysical (as in the case of the Schwarzschild black string), it is interesting to explore the properties of a brane with collapsing $\mathrm{KK}$ energy density, since this idealized toy model may lead to important physical insights into more realistic collapse with matter and KK energy density.

The exterior is static and unique, and given by the Weylcharged de Sitter metric

$$
d s^{2}=-A d t^{2}+d R^{2} / A+R^{2} d \Omega^{2}, \quad M=0,
$$

if $A>0$. For $Q=0$ it is de Sitter, with horizon $H^{-1}=$ $\sqrt{3 / \Lambda}$. For $\Lambda=0$ it is the special case $M=0$ of the solutions given in [6], and the length scale $H_{Q}^{-1}=\sqrt{|Q| / \lambda}$ is a horizon when $Q>0$; for $Q<0$, there is no horizon. As we show below, the interplay between these scales determines the characteristics of collapse.

For $\Lambda=0$, the exterior gravitational potential is

$$
\Phi=Q / 2 \lambda R^{2},
$$

which has the form of a purely 5D potential when $Q>0$. When $Q<0$, the gravitational force is repulsive. We thus take $Q>0$ as the physically more interesting case, corresponding to positive KK energy density in the interior. However, we note the remarkable feature that $Q>0$ also implies negative $\mathrm{KK}$ energy density in the exterior:

$$
-\mathcal{E}_{\mu \nu} u^{\mu} u^{\nu}= \begin{cases}+3 Q /\left(\lambda R_{\Sigma}^{4}\right), & R<R_{\Sigma}, \\ -Q /\left(\lambda R^{4}\right), & R>R_{\Sigma} .\end{cases}
$$

Negativity of the exterior KK energy density in the general case with matter has been previously noted $[4,6]$.

The boundary surface between the KK "cloud" and the exterior has equation of motion $\dot{R}^{2}=E-V(R)$, where $V=A-1$. For $\Lambda=0$, the cases are as follows:

$Q>0, \Lambda=0$ : The cloud collapses for all $E$, with horizon at $R_{\mathrm{h}}=H_{Q}^{-1}=\sqrt{Q / \lambda}$. For $E<0$, given that $E>-1$, the collapse can at most start from rest at $R_{\max }=$ $\sqrt{Q /(\lambda|E|)}>R_{\mathrm{h}}$.

$Q<0, \Lambda=0$ : It follows that $E>0$, there is no horizon, and the cloud bounces at $R_{\min }=\sqrt{|Q| /(\lambda E)}$.

For $\Lambda>0$, the potential is given by $V / V_{\mathrm{c}}=$ $-\left(R / R_{\mathrm{c}}\right)^{2}\left[1+\epsilon\left(R_{\mathrm{c}} / R\right)^{4}\right]$, where $V_{\mathrm{c}}=H / H_{Q}, R_{\mathrm{c}}=1 /$ $\sqrt{H H_{Q}}$, and $\epsilon=\operatorname{sgn} Q$ (see Fig. 1). The horizons are given by

$$
\left(R_{\mathrm{h}}^{ \pm}\right)^{2}=R_{\mathrm{c}}^{2}\left[1 \pm \sqrt{1-4 \epsilon V_{\mathrm{c}}^{2}}\right] / 2 V_{\mathrm{c}} .
$$

If $\epsilon>0$ there may be two horizons; then $R_{\mathrm{h}}^{-}$is the black hole horizon and $R_{\mathrm{h}}^{+}$is a modified de Sitter horizon. When they coincide the exterior is no longer static, but there is a black hole horizon. If $\epsilon<0$ there is always one de Sitterlike horizon, $R_{\mathrm{h}}^{+}$.

$Q>0, \Lambda>0$ : The potential has a maximum $-2 V_{\mathrm{c}}$ at $R_{\mathrm{c}}$. If $E>-2 V_{\mathrm{c}}$ the cloud collapses to a singularity. 


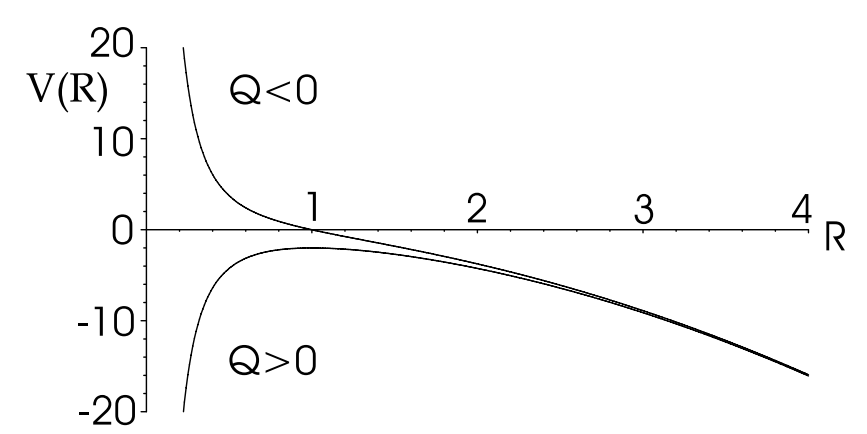

FIG. 1. The potential $V(R)$ for $\Lambda>0$, with $R$ given in units of $R_{\mathrm{c}}$ and $V$ given in units of $V_{\mathrm{c}}$.

For $V_{\mathrm{c}}>\frac{1}{2}$, i.e., $Q>3 \lambda / 4 \Lambda$, there is no horizon, and a naked singularity forms. For $V_{\mathrm{c}}=\frac{1}{2}$ there is one black hole horizon $R_{h}^{-}=R_{h}^{+}=H^{-1} / \sqrt{2}$. If $E \leq-2 V_{\mathrm{c}}$, then Eq. (8) implies $V_{\mathrm{c}}<\frac{1}{2}$, so there are always two horizons in this case. Either the cloud collapses from infinity down to $R_{\min }$ and bounces, with $R_{\min }<R_{\mathrm{h}}^{+}$always, or it can at most start from rest at $R_{\max }\left(>R_{\mathrm{h}}^{-}\right)$, and collapses to a black hole, where $(\epsilon=1)$

$$
R_{\max }^{2}=R_{\mathrm{c}}^{2}\left[-E \pm \sqrt{E^{2}-4 \epsilon V_{\mathrm{c}}^{2}}\right] / 2 V_{\mathrm{c}} .
$$

$Q<0, \Lambda>0$ : The potential is monotonically decreasing, and there is always a horizon, $R_{\mathrm{h}}^{+}$. For all $E$, the cloud collapses to $R_{\min }\left(<R_{\mathrm{h}}^{+}\right)$and then bounces, where $R_{\min }$ is given by Eq. (19) with $\epsilon=-1$.

In summary, we have explored the consequences for gravitational collapse of braneworld gravity effects, using the simplest possible model, i.e., an OS-like collapse on a generalized Randall-Sundrum-type brane. Even in this simplest case, extra-dimensional gravity introduces new features. Using only the projected 4D equations, we have shown, independent of the nature of the bulk, that the exterior vacuum on the brane is necessarily nonstatic. This contrasts strongly with GR, where the exterior is a static Schwarzschild spacetime. Although we have not found the exterior metric, we know that its nonstatic nature arises from (a) 5D bulk graviton stresses, which transmit effects nonlocally from the interior to the exterior and (b) the nonvanishing of the effective pressure at the boundary, which means that dynamical information on the interior side can be conveyed outside. Our results suggest that gravitational collapse on the brane may leave a signature in the exterior, dependent upon the dynamics of collapse, so that astrophysical black holes on the brane may in principle have KK hair.

We expect that the nonstatic exterior will be transient and nonradiative, as follows from a perturbative study of nonstatic compact objects, showing that the Weyl term $\mathcal{E}_{\mu \nu}$ in the far-field region falls off much more rapidly than a radiative term [4]. It is reasonable to assume that the exterior metric will be static at late times and tend to Schwarzschild, at least at large distances.

We have analyzed the idealized collapse of homogeneous KK energy density whose exterior is static and has purely 5D gravitational potential. The collapse can either come to a halt and bounce or form a black hole or a naked singularity, depending on the parameter values. This may be seen as a limiting idealization of a more general spherically symmetric but inhomogeneous case. The case that includes matter may be relevant to the formation of primordial black holes in which nonlinear KK energy density could play an important role.

C. G. is supported by PPARC. M. B. and R. M. thank the Mathematical Cosmology Programme at ESI, Vienna, where part of this work was done.

[1] H. Stephani, General Relativity (Cambridge University Press, Cambridge, 1990).

[2] L. Randall and R. Sundrum, Phys. Rev. Lett. 83, 4690 (1999).

[3] T. Shiromizu, K. I. Maeda, and M. Sasaki, Phys. Rev. D 62, 024012 (2000).

[4] M. Sasaki, T. Shiromizu, and K. I. Maeda, Phys. Rev. D 62, 024008 (2000).

[5] J. Garriga and T. Tanaka, Phys. Rev. Lett. 84, 2778 (2000); C. Csaki, J. Erlich, T. J. Hollowood, and Y. Shirman, Nucl. Phys. B581, 309 (2000); S. B. Giddings, E. Katz, and L. Randall, J. High Energy Phys. 03, 023 (2000); I. Ya. Araf'eva, M. G. Ivanov, W. Muck, K. S. Viswanathan, and I. V. Volovich, Nucl. Phys. B590, 273 (2000); Z. Kakushadze, Phys. Lett. B 497, 125 (2000); I. Giannakis and H.C. Ren, Phys. Rev. D 63, 024001 (2001); N. Deruelle and T. Dolezel, Phys. Rev. D 64, 103506 (2001).

[6] N. Dadhich, R. Maartens, P. Papadopoulos, and V. Rezania, Phys. Lett. B 487, 1 (2000).

[7] C. Germani and R. Maartens, hep-th/0107011 [Phys. Rev. D (to be published)].

[8] A. Chamblin, S.W. Hawking, and H.S. Reall, Phys. Rev. D 61, 065007 (2000).

[9] T. Shiromizu and M. Shibata, Phys. Rev. D 62, 127502 (2000); A. Chamblin, H.S. Reall, H. A. Shinkai, and T. Shiromizu, Phys. Rev. D 63, 064015 (2001).

[10] R. Maartens, Phys. Rev. D 62, 084023 (2000); gr-qc/ 0101059 .

[11] S. Mukohyama, T. Shiromizu, and K. I. Maeda, Phys. Rev. D 62, 024028 (2000).

[12] P. Bowcock, C. Charmousis, and R. Gregory, Classical Quantum Gravity 17, 4745 (2000).

[13] P. Binetruy, C. Deffayet, U. Ellwanger, and D. Langlois, Phys. Lett. B 477, 285 (2000).

[14] M. M. Prats, Ph.D. thesis, University of Barcelona, 1995. 